\title{
Oseltamivir-resistant pandemic H1N1 influenza
}

\author{
Duncan Webster MA MD, Yan Li PhD, Nathalie Bastien PhD, Richard Garceau MD, Todd F. Hatchette MD
}

Competing interests:

None declared.

This article has been peer reviewed.

Correspondence to: Dr. Duncan Webster, duncan.webster@horizon NB.ca

CMAJ 2011. DOI:10.1503 /cmaj.100313
I n December 2009, a 27-year-old man with severe influenza-like illness was admitted to a tertiary care hospital in Saint John, New Brunswick. The patient had asthma, obesity (body mass index $40.7 \mathrm{~kg} / \mathrm{m}^{2}$ ) and untreated familial hypercholesterolemia. He had not received immunization against pandemic $\mathrm{H} 1 \mathrm{~N} 1$ influenza. He had been prescribed oral oseltamivir $75 \mathrm{mg}$ twice daily the previous week for presumed pandemic H1N1 infection, but had discontinued the medication after four days because of adverse gastrointestinal effects. The patient's condition deteriorated rapidly after admission, and he was received mechanical ventilation.

Oseltamivir $150 \mathrm{mg}$ twice daily was started in combination with moxifloxacin. Although the nasopharyngeal aspirate was negative, the endotracheal secretions were positive for pandemic H1N1 on a reverse transcriptase polymerase chain reaction (RT-PCR) test. The patient did not improve, and his antibacterial agent was switched to piperacillin-tazobactam. Results of a repeat RTPCR test of endotracheal secretions remained positive after 12 days in hospital. The patient was given intravenous zanamivir for presumed resistance to oseltamivir that was later confirmed by laboratory testing. Despite initial stabilization after completion of the five-day course of zanamivir, cardiac ischemia developed and the patient died three weeks after admission. Brain, heart and lung specimens obtained at autopsy were negative for pandemic H1N1 on RT-PCR testing.

\section{KEY POINTS}

- Immunization of people at high risk for severe influenza illness is important for primary prevention.

- When patients fail to show clinical improvement despite appropriate first-line antiviral treatment, repeat testing for influenza, using reversetranscriptase polymerase chain reaction, and testing for resistance to antiviral agents should be considered.

- For patients receiving mechanical ventilation, deep endotracheal suction specimens should be obtained for influenza testing in addition to nasopharyngeal swabs.

- When viral excretion persists and patients fail to show clinical improvement despite oseltamivir therapy, switching to another antiviral agent should be considered.

\section{Discussion}

As of May 1, 2010, a total of 8678 instances of patients admitted to hospital with pandemic H1N1 had been documented in Canada since the beginning of the pandemic in April 2009. ${ }^{1}$ This number included 1473 cases (17.0\%) requiring admission to intensive care units across the country, with death observed in $4.9 \%$ of instances $(n=428)$. The presence of comorbidities has been associated with severe illness and a mortality 25.5 times higher than among patients without underlying medical conditions. Chronic pulmonary disease, including asthma, has been the most commonly reported underlying medical condition, observed in 51.3\% of deaths. Throughout the H1N1 pandemic, neuraminadase inhibitors were recommended for treatment of severe illness caused by the pandemic $\mathrm{H} 1 \mathrm{~N} 1$ virus, as well as for selective postexposure prophylaxis in high-risk people. ${ }^{2,3}$ Though rare, resistance to oseltamivir has emerged in many countries, and it has been hypothesized that resistance could increase over time as the drug is used extensively on a global scale. ${ }^{4}$ As of Jan. 26, 2011, 340 instances of oseltamivir resistance had been reported by the World Health Organization Global Influenza Surveillance Network. ${ }^{5}$

\section{Neuroaminadase inhibitors}

During the final stages of the influenza replication cycle, newly assembled viral particles bud from the host cell. The viral enzyme neuraminidase cleaves the attachment, allowing the new viruses to be released so that they may infect new host cells. Oseltamivir is a neuraminidase inhibitor, which acts by preventing this cleavage step, thus interfering with the release of progeny virus and preventing the progression of infection (Figure 1).

With the H275Y mutation, a conformational change occurs at the binding site of the neuraminidase inhibitor, preventing binding of oseltamivir. Hence, the new virus is not impeded from completing its replication cycle and may undergo cleavage and release from the host cell. In $26 \%$ of reported instances of oseltamivir resis- 
tance, there has been an association with treatment, whereas $6 \%$ have been associated with postexposure prophylaxis. ${ }^{6}$ During the course of the pandemic, millions of doses of oseltamivir were administered worldwide, yet only 340 instances of oseltamivir resistance have been identified. ${ }^{5}$ In Canada by Feb. 4, 2010, more than 800 isolates were tested, and only 12 were found to be oseltamivir-resistant (Y.L. and N.B., unpublished data, 2010.) To date, all but one of these oseltamivir-resistant strains of pandemic H1N1 have been found to have the H275Y mutation. Resistance is infrequent and detected sporadically in pandemic $\mathrm{H} 1 \mathrm{~N} 1$, although the incidence of oseltamivir resistance is likely underreported because relatively few viruses are tested. There are currently no data to suggest that longer courses of higher doses would prevent resistance from developing.

\section{Predicting resistance}

It is very difficult to predict when drug resistance will develop. The National Microbiology Laboratory has provided protocols to provincial public health laboratories to facilitate rapid testing for the H275Y mutation. ${ }^{7}$ Guidance published by the Canadian Public Health Laboratory Network ${ }^{8}$ suggests clinicians should suspect resistance to antiviral medication when influenza is detected in patients receiving prophylaxis, when infection persists in immunocompromised hosts, in instances in which patients have had contact with immunocompromised hosts undergoing treatment, and in patients whose condition fails to improve with oseltamivir therapy.

The definition of clinical treatment failure in pandemic H1N1 infection has not been established. A study involving patients infected with the $\mathrm{H} 5 \mathrm{~N} 1$ virus showed that treatment failure was

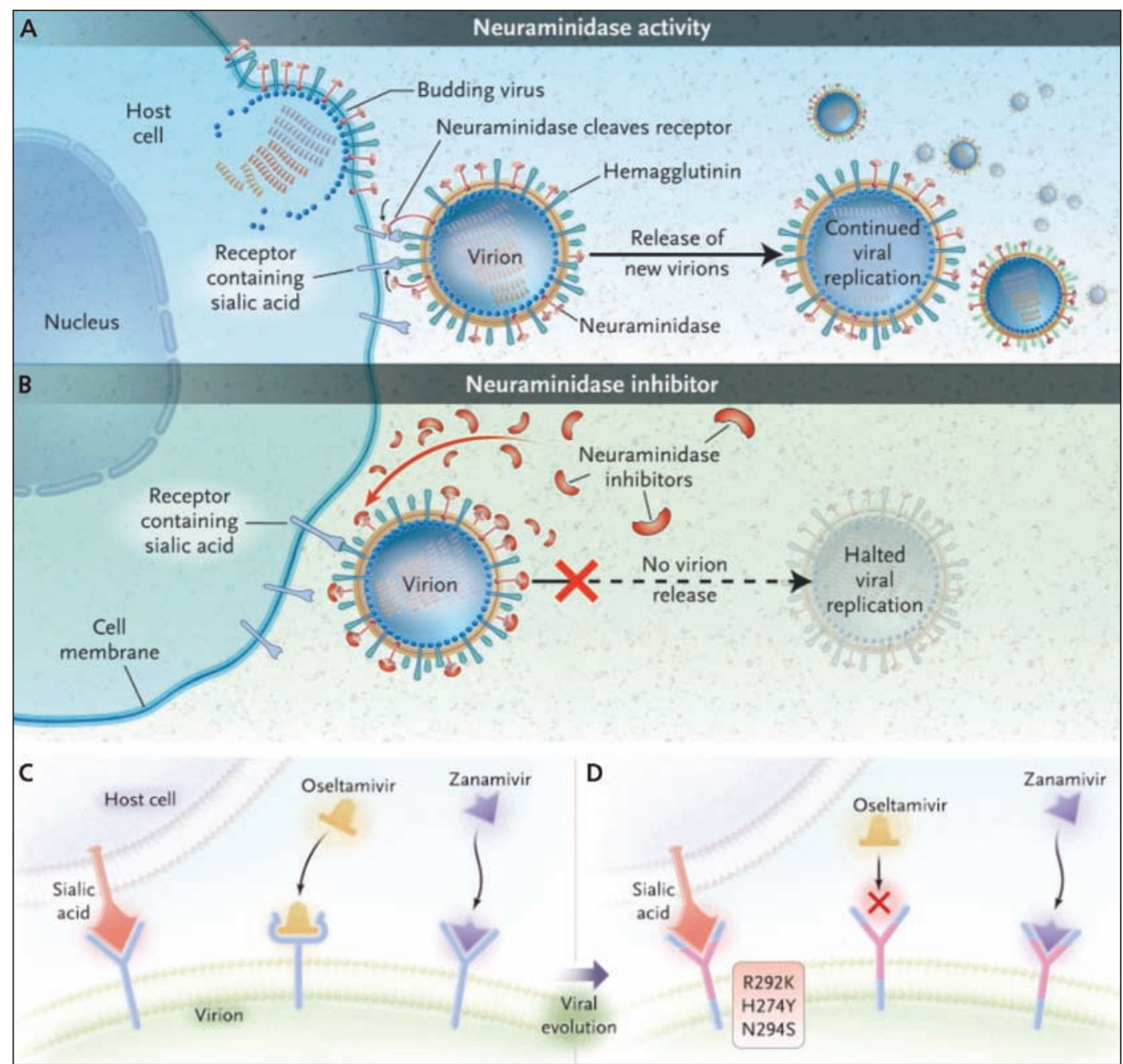

Figure 1: (A) The action of neuraminidase in the continued replication of virions in the setting of influenza infection. The replication is blocked by neuraminidase inhibitors (B), which prevent virions from being released from the surface of infected cells. (C) Binding of oseltamivir and zanamivir to the neuraminidase-active sites. (D) With mutation, a conformational change at the binding site prevents binding of oseltamivir but permits binding of zanamivir. (Images obtained from N Engl J Med 2005;353:1363-73 and N Engl J Med 2005;353:2633-6 and reprinted with permission of the publisher. Copyright $\odot 2005$ Massachusetts Medical Society. All rights reserved.) 
associated with persistent high viral load after 48 hours of therapy. ${ }^{9}$ However, viral load testing is not available routinely. For practical purposes, patients who continue to deteriorate with no other identifiable cause despite 10 days of oseltamivir treatment should be tested for resistance. ${ }^{8}$

\section{Alternative treatments}

The H275Y mutation confers resistance to oseltamivir, but susceptibility is preserved to zanamivir, a neuraminidase inhibitor commercially available for use via inhalation (Figure 1). However, the use of zanamivir is limited by the fact that it is an inhaled compound that cannot be administered to ventilated patients, and the intravenous formulation can be obtained in Canada only through the Special Access Program of Health Canada. Until there are more readily available intravenous antiviral medications, treatment options are limited, and clinicians should consider seeking advice from a colleague specializing in infectious diseases or medical microbiology. If the patient is able to take inhaled zanamivir, it may be reasonable to change antiviral medications pending resistance testing.

\section{Minimizing resistance}

Notably, the development and maintenance of oseltamivir resistance in seasonal influenza viruses occurred in the absence of substantial global use; the reason is unclear. To minimize the risk of development of resistance, antiviral agents should be used appropriately and at adequate doses according to published guidelines. This recommendation may be particularly relevant to the pediatric and immunocompromised populations, in which the risk of generating resistance is greatest. ${ }^{10}$

The pandemic H1N1 virus will likely evolve into a "seasonal" influenza A strain in the coming years. Indeed, the previous "seasonal" H1N1 strain circulating prior to the pandemic has virtually disappeared across the globe. ${ }^{11}$ To date, the predominant circulating strain in Canada this

\section{Resources for clinicians}

\section{Treatment recommendations}

- The use of antiviral medications for influenza: guidance for practitioners, 2010-11. www.ammi.ca/pdf/UseOfAntiviralDrugs.pdf

- Antiviral agents for the treatment and chemoprophylaxis of influenza: recommendations of the advisory committee on immunization practices (ACIP). www.cdc.gov/mmwr/pdf/rr/rr6001.pdf

\section{Laboratory testing guidelines}

- Guidance for laboratory testing for detection and characterization of human influenza virus for the 2010-2011 respiratory virus season: www.cphln.ca /documents/EN_influenza_seasonal_best_practices_2010-2011.pdf season has been the A/Perth/6/2009-like H3N2 virus. Although pandemic-H1N1-like virus accounts for only $10.5 \%$ of influenza viruses subtyped thus far in Canada, it is the dominant strain currently circulating in the United Kingdom and other parts of Europe..$^{12}$ Because strains containing the $\mathrm{H} 275 \mathrm{Y}$ mutation encoding oseltamivir resistance are antigenically similar to the vaccine strain, vaccination will most likely continue to be the primary method to reduce the burden of disease caused by this and other new strains.

\section{References}

1. Public Health Agency of Canada. FluWatch: April 25 to May 1, 2010 (Week 17). Ottawa (ON): The Agency; 2010. Available: www.phac-aspc.gc.ca/fluwatch/09-10/w17_10/index-eng.php (accessed 2010 June 29)

2. Centers for Disease Control and Prevention. Updated interim recommendations for the use of antiviral medications in the treatment and prevention of influenza for the 2009-2010 season. Atlanta (GA): The Center; 2010. Available: www.cdc.gov /h1n1flu/recommendations.htm (accessed 2010 June 7).

3. Public Health Agency of Canada. Clinical recommendations for patients presenting with respiratory symptoms during the 20092010 influenza season. Ottawa (ON): The Agency; 2010. Available: www.phac-aspc.gc.ca/alert-alerte/h1n1/pdf/H1N1 _DecisionTree_oct23_e.pdf (accessed 2010 June 7).

4. Baum SG. Oseltamivir-resistance: What does it mean clinically? Clin Infect Dis 2009;49:1836-7.

5. World Health Organization. Weekly update on oseltamivir resistance to influenza A (HIN1) 2009 viruses. Geneva: The Organization; 2010. Available: www.who.int/csr/disease/influenza /2011_01_28_weekly_web_update_oseltamivir_resistance.pdf (accessed 2011 Feb. 5).

6. Baz M, Abed Y, Papenburg J, et al. Emergence of oseltamivirresistant pandemic H1N1 virus during prophylaxis. $N$ Engl J Med 2009;361:2296-7.

7. Canadian Public Health Laboratory Network. Interim guidance for laboratory testing for detection and characterization of pandemic HINI (2009) virus. Winnipeg (MB): The Network; 2009. Available: www.cphln.ca/pubs_best_practices_eng.html (accessed 2010 June 8).

8. Canadian Public Health Laboratory Network. Guidance for laboratory testing for detection and characterization of human influenza virus for the 2010-2011 respiratory virus season. Winnipeg (MB): The Network; 2010. Available: www.cphln.ca /documents/EN_influenza_seasonal_best_practices_2010-2011.pdf (accessed 2011 Feb. 5).

9. de Jong MD, Tran TT, Truong HK, et al. Oseltamivir resistance during treatment of influenza A (H5N1) infection. $N$ Engl J Med 2005;353:2667-72.

10. Moscona A. Oseltamivir resistance - disabling our influenza defences. N Engl J Med 2005;353:2633-6.

11. World Health Organization. Global circulation of influenza viruses. Geneva: The Organization; 2011. Available: www.who.int /csr/disease/influenza/2011_01_28_influenza_global_circulation .pdf (accessed 2011 Feb. 4).

12. Public Health Agency of Canada. FluWatch: January 16 to 22, 2011 (Week 03). Ottawa (ON): The Agency; 2011. Available: www.phac-aspc.gc.ca/fluwatch/10-11/w03_11/pdf/fw2011-03-eng .pdf (accessed 2011 Feb. 4).

Affiliations: From the Department of Medicine (Webster), Saint John Regional Hospital, Saint John, NB; the Department of Pathology and Laboratory Medicine (Hatchette), Dalhousie University, Halifax, NS; the National Microbiology Laboratory (Bastien, Li), Public Health Agency of Canada, Winnipeg, Man.; and the Department of Medical Microbiology (Garceau), Hôpital Dr. G.L. Dumont, Moncton, NB

Contributors: All of the authors were involved in the conception and design of the article, the acquisition or analysis and interpretation of the data, and the drafting and revision of the manuscript. All of them approved the final version submitted for publication. 